\title{
MOFs as hosts to analogues of complex magnetic phases
}

\author{
Emma H. Wolpert ${ }^{1}$, François-Xavier Coudert ${ }^{2}$, Andrew L. Goodwin ${ }^{1}$
}

\author{
${ }^{1}$ University of Oxford, U.K. \\ ${ }^{2}$ Université Paris Sciences et Lettres, France \\ e.wolpert@imperial.ac.uk
}

Controlling the mesoscopic nature of materials through local interactions can lead to the formation of highly non-topologically trivial structures. The local interactions that lead to the emergence of mesoscopic structures, known as textures, is well understood in magnetic materials. The most studied textures are skyrmions as the have interesting applications in spintronics due to their topological nature and dynamic properties [1]. These features are thought to be exclusively a magnetic characteristic; however, they are purely topological in nature and arise due to a specific set of interactions which may not be limited to magnetic materials. As we have an increased understanding of what causes topological properties, we can design/search for specific interactions in non-magnetic materials that may lead to non-magnetic analogues of topologically protected phases.

For skyrmions to exist, three interactions must be present: symmetric exchange, antisymmetric exchange, and a coupling to a magnetic field [2]. To explore the possibility of creating analogues of magnetic textures in non-magnetic materials we replace magnetic dipoles with non-magnetic quadrupoles and exchange the magnetic field for a strain field and adapt the interactions accordingly. Here we look at the capability of MOFs to harbor analogous complex magnetic phases such as skyrmions. MOFs are the perfect candidates as there are a plethora of components to play with such as underlying lattice, guest species, and interactions between the framework and the guest.

Through density functional theory calculations, molecular dynamics simulations, and Monte Carlo simulations, we explore the extent to which these interactions may exist in chiral MOF frameworks with quadrupolar guests such as a benzene or $\mathrm{CO}_{2}$ and how varying the relative strengths of the three interaction parameters with temperature effects the behaviour of the non-magnetic textures. Using small angle scattering we have been able to define six distinct phases, giving evidence of quadrupolar skyrmions and interesting textures which are not present in the dipolar analogue. This opens up the field to new ways of creating non-topologically trivial textures that could potentially be less restrictive than chiral magnets.

[1] S. Mühlbauer, B. Binz, F. Jonietz, C. Pfleiderer, A. Rosch, A. Neubauer, R. Georgii, and P. Boni, Science 323, 915 (2009).

[2] S. D. Yi, S. Onoda, N. Nagaosa, and J. H. Han, Phys. Rev. B. 80, 054416 (2009).

Keywords: Topology, mapping, metal organic frameworks, guest host interactions 